\title{
DE KANT A KUHN, ACOTANDO POR PUTNAM*
}

\author{
Ana Rosa PÉREZ RANSANZ \\ UNAM \\ José Francisco ÁLVAREZ \\ UNED
}

ABSTRACT: Our aim in this paper is to trace in some detail the deep influence of Kant's epistemology on T.S. Kuhn's model of scientific change, specially on the kind of realism this model presupposes. In order to do this, we highlight the hard core thesis of what H. Putnam (1981) has called "internal realism" - a kind of kantian realismand then we try to show the strong resemblance between these thesis and Kubn's ontological commitments. Last, we point to some advantages this kantian perspective offers in the way to construct a more adequate notion of objectivity and rationality.

Las siguientes reflexiones tratan de mostrar algunos acuerdos básicos que subyacen a dos concepciones del conocimiento que, aunque lejanas en el tiempo, han representado, cada una en su momento, una revolución en el ámbito filosófico: la epistemología kantiana y el modelo kuhniano de la dinámica científica. Estos acuerdos se rastrean en torno a la relación entre conocimiento y mundo, relación cuyas interpretaciones diversas han ido marcando los derroteros en el debate sobre el realismo y la noción de verdad - y en particular, en la discusión sobre el realismo en la ciencia.

Con excesiva frecuencia, quienes tienen en cuenta a Kant desde la filosofía contemporánea de la ciencia no llegan, en el mejor de los casos, sino a localizar la fuente de la archicitada expresión de Lakatos «La filosofía de la ciencia sin la historia de la ciencia está vacía; la historia de la ciencia sin la filosofía de la ciencia está ciega" (Lakatos 1971, p. 455). La famosa sentencia de Kant aparece en

${ }^{1}$ El presente trabajo es fruto de la actividad conjunta realizada en el marco del proyecto «Capacidades potenciales, racionales acotada y evaluación tecnocientífica" (UNED-UNAM) subvencionado por la AECI para el año 2003 dentro del Programa de Cooperación Científica con Iberoamérica. 
La critica de la razón pura, en su Introducción a la Lógica Trascendental, y reza así: "Los pensamientos sin contenidos son vacíos; las intuiciones sin conceptos son ciegas", pero continúa: «Por ello es tan necesario hacer sensibles los conceptos (es decir, añadirles el objeto en la intuición) como hacer inteligibles las intuiciones (es decir, someterlas a conceptos)" (KRV, B 75). Intentaremos enfocarnos un poco más en la obra de Kant y ver hasta qué punto sus consideraciones epistémicas están presentes en la filosofía de la ciencia en una medida mayor de lo que permite atisbar una primera mirada, sesgada por la separación en especialidades filosóficas.

A nuestro juicio, la concepción del conocimiento que presupone el modelo de Thomas Kuhn no sólo resulta compatible con un realismo de tipo interno, esto es, un realismo de raigambre kantiana, sino que además dicha concepción nos permitiría actualizar este tipo de realismo y destacar su engarce con una noción más adecuada de racionalidad, al aportar elementos que provienen de un análisis innovador del modo como la ciencia cambia y evoluciona.

En la arena del debate sobre el realismo, los desacuerdos entre los filósofos de la ciencia no se reducen - como piensan algunos- a las discrepancias sobre "qué tanto de un buen modelo corresponde a la realidad" (Cf. van Fraassen 1989, p. 188). Más bien, creemos, los desacuerdos básicos se generan en la manera de entender la relación de correspondencia o de representación entre teoría y realidad, así como en la manera de concebir cada uno de los polos de esta relación. En definitiva, los temas del realismo y su conexión con la epistemología tienen que ver más con la naturaleza de las relaciones que con la de las entidades; por decirlo de manera concisa, el problema hace referencia a predicados de orden estrictamente superior a los monádicos.

De aquí que con miras a catalogar el compromiso realista de Kuhn dentro de la familia de realismos de cuńo kantiano, debamos primero examinar aquello que, a nuestro juicio, constituye el núcleo duro de una perspectiva filosófica como la que Hilary Putnam (1981) denominara «internalista», rastreando su fuerte anclaje en el pensamiento de Kant. Dicho núcleo constaría de dos tesis fundamentales: la primera, la que establece la noción de objeto como objeto conceptualmente constituido; la segunda, la que rechaza la idea de verdad como correspondencia entre nuestros juicios empíricos y la realidad independiente de nuestro conocimiento. A la luz de estas tesis, que implican una clara oposición al realismo metafísico, se puede entender que muchos autores prefieran catalogar al 
realismo interno -o internalista- como otra forma más de antirrealismo. Sin embargo pretendemos decir que, después de la crítica kantiana, parece indispensable tomarse en serio nuestra capacidad como sujetos productores de conocimiento y que, dada nuestra constitución como sujetos epistémicos, a lo más que podemos aspirar es a sostener un coherente realismo interno fuente de la objetividad humanamente posible, aquélla que pueden alcanzar sujetos que no son dioses del Olimpo. Lo que en nuestros tiempos suele llamarse conocimiento encarnado se conecta con esa línea que pasa por Kant y Kuhn.

\section{Kant, Putnan y la realidad independiente}

Comencemos entonces con la discusión sobre la noción de objeto, la cual nos remite al viejo problema de si hay "algo dado", independiente del conocimiento. Como adelantamos, estas cuestiones son medulares en tanto que su respuesta apunta a la raíz de las discrepancias entre realistas y antirrealistas, así como a la de las diferencias entre los distintos tipos de realismo.

De entrada digamos que el propio Putnam, el de los años 80 (precisión necesaria pues es bien sabido que él mismo se divierte con sus constantes cambios de posición y cómo ello perturba a sus acólitos), reconoce que su enfoque está muy cerca de la filosofía de Kant; e incluso afirma - en esa misma década - que la mejor manera de leer a este clásico es como si propusiera, por primera vez, un realismo interno (Cf. Putnam 1981, p. 60). De aquí la conveniencia de seguir paso a paso su análisis de las tesis kantianas.

Este análisis comienza con la sugerencia de que, como primera aproximación, se lea a Kant como un generalizador de lo que Locke afirmó sobre las cualidades secundarias — color, textura, sabor, etc.- tratando de aplicarlo a todas las cualidades o propiedades de los objetos. Esto implicaría que todo lo que dijéramos acerca de un objeto sería de la forma: el objeto es de tal naturaleza que nos afecta de tal y cual manera. Por tanto, nada de lo que pudiéramos decir de un objeto lo describiría tal como es "en sí mismo», independientemente de su efecto en nosotros, es decir, en seres con nuestra naturaleza racional y nuestra constitución biológica. Como se puede suponer, esta lectura pondría de relieve, sin mayor rodeo, la perspectiva internalista de Kant. Además, la idea de que todas las propiedades son secundarias implicaría también que no tenemos bases 
para afirmar ninguna similitud entre nuestra idea de un objeto y aquello que en la realidad independiente pudiera ser responsable de nuestra experiencia de dicho objeto (Cf. ibid., pp. 60-61).

Ahora bien, es importante señalar desde un principio que Kant nunca pone en duda la existencia de una realidad independiente de la mente; para él, esto es un postulado de la razón. Sin embargo, su interés consiste en destacar que, a pesar de ello, no nos podemos formar ninguna idea o concepto de los componentes de dicha realidad, es decir, de los "noúmenos" o "cosas-en-sín. Incluso se podría afirmar que la noción de noúmeno, si bien puede tener cierto sentido formal, es más bien un concepto negativo que alude a lo incognoscible por definición, como tal sólo cumple el papel de señalar un límite infranqueable de nuestra experiencia. Por tanto, hablar de objetos empíricos es siempre hablar de cosas-para-nosotros, nunca de cosas-en-sí. En este mismo orden de cuestiones, otro aspecto importante, que distingue a Kant de la tradición, es que estas tesis las aplica por igual tanto a los "objetos externos" como a los "objetos del sentido interno", es decir, tanto a las cosas físicas como a las entidades mentales, lo cual significaría que estas últimas no están más cerca de lo nouménico de lo que lo están los objetos físicos o materiales.

Pero examinemos la razón de que la sugerencia de leer a Kant como si afirmara que todas las propiedades son secundarias, funcione sólo como una primera aproximación, no del todo adecuada, a pesar de su valor heurístico. Según Putnam, el hecho de que cierta propiedad de un objeto sea secundaria - como el "ser rojo" de un pedazo de tela - se explicaría por una propiedad disposicional compleja del objeto, o en términos de Locke, por un "poder» que éste tiene de producir una sensación de cierto tipo, bajo determinadas circunstancias. "Este poder a su vez tiene una explicación -que no se conocía en tiempos de Locke- en la microestructura particular del pedazo de tela, la cual hace que ésta selectivamente absorba y refleje luz de diferentes longitudes de onda" (Ibíd., p. 58). De aquí que resulte absurdo suponer que la propiedad de la representación, o imagen mental, es literalmente la misma que la propiedad del objeto físico. (Aunque cabría recordar que para Locke, en el caso de las cualidades primarias como movimiento, forma, posición, etc., sí existía una semejanza literal entre la idea y el objeto representado.)

De esta manera, cuando se afirma "todas las propiedades son secundarias" iría implícito algo que Kant no hubiera aceptado. Formulado en términos de 
Locke, decir de una silla que es de color azul es atribuirle a un objeto nouménico la disposición de aparecer azul ante nosotros; decir de la silla que está hecha de pino es atribuirle una disposición diferente al mismo objeto nouménico, y así sucesivamente. Por tanto, bajo esta concepción, habría un objeto nouménico, una cosa-en-sí, que correspondería a cada cosa-para-nosotros; es decir, habría una especie de correspondencia biunívoca entre cosas-en-sí y cosas-para-nosotros. Pero como afirma Putnam - y nosotros suscribimos convencidos- la perspectiva kantiana excluiría esta posibilidad, puesto que eso supondría dar por resuelto el problema mismo que Kant trataba de resolver. Para establecer aquel posible tipo de correspondencia tendríamos que tener un acceso a la cosa-en-sí con independencia de la cosa-para-nosotros. Y si bien debemos reconocer que con frecuencia Kant utiliza el plural con respecto a lo en-sí (cosas-en-sí o noúmenos), lo importante es que de hecho abandona toda idea de semejanza o similitud entre nuestras ideas y las cosas-en-sí, descartando con ello cualquier tipo de isomorfismo o correspondencia estricta entre realidad independiente y mundo para nosotros, entre mundo nouménico y mundo fenoménico.

Por otra parte, aunque también es cierto que Kant asume la idea de verdad como "correspondencia de un juicio con su objeto", sin embargo sería un grave error identificar dicha correspondencia con la correspondencia metafísica que sustenta la teoría de la verdad defendida por los externalistas. Pero para entender correctamente esta "definición nominal de la verdad", como la llama Kant, es necesario elucidar el papel que cumple el mundo nouménico en un juicio empírico. Dice Putnam: «En la concepción kantiana, todo juicio acerca de objetos externos o internos (cosas físicas o entidades mentales) dice que el mundo nouménico, como un todo, es tal que ésta es la descripción que construiría un ser racional (uno con nuestra naturaleza racional), dada la información disponible para un ser con nuestros órganos sensoriales (un ser con nuestra naturaleza sensible)» (Ibid., p. 63).

Notemos entonces que el mundo nouménico juega un papel en la descripción de objetos empíricos - y en general en la construcción del conocimientosólo en la medida en que se le considera como un todo. Es en tanto totalidad, como un algo indeterminado para nosotros, que se le atribuye un poder a esa realidad independiente de que los juicios empíricos sean como son, es decir, que sean el tipo de descripción de objetos que tendría que construir un ser con nuestra naturaleza racional y sensible, dada la información y los recursos disponibles. De aquí que la forma en que ese algo independiente posibilita nuestro conoci- 
miento de objetos no pueda concebirse como una relación uno-a-uno entre cosas-en-sí y cosas-para-nosotros: «no se debe pensar que porque hay sillas y caballos y sensaciones en nuestra representación, hay correspondientemente sillas nouménicas y caballos nouménicos y sensaciones nouménicas» (Ibidem).

La interpretación que se hace de Kant como alguien que afirma la existencia de una realidad nouménica isomorfa al mundo de objetos empíricos, encierra una idea asaz frecuente en las posiciones realistas de corte metafísico, idea que precisamente nos remite a la manera de entender lo que son los objetos. Por tanto, dejemos por el momento de lado la lectura que hace Putnam de Kant, para adentrarnos en el análisis de la idea kantiana de objeto, así como en las ideas conexas de existencia y realidad.

Al intentar precisar su noción de objeto en general, Kant afirma: «Los fenómenos son los únicos objetos que se nos pueden dar inmediatamente y lo que en ellos hace referencia inmediata al objeto se llama intuición. Pero tales fenómenos no son cosas en sí mismas, sino meras representaciones que, a su vez, poseen su propio objeto, un objeto que ya no puede ser intuido por nosotros y que, consiguientemente, puede llamarse no-empírico, es decir, trascendental = X. El concepto puro de este objeto trascendental (que, de hecho, es idéntico en todos nuestros conocimientos, $=\mathrm{X}$ ) es lo que pone en relación todos nuestros conceptos empíricos con un objeto, es decir, lo que les puede suministrar realidad objetiva" (KRV, A 108-109; énfasis nuestro). En esta cita encontramos, claramente, dos nociones de objeto. Por un lado, la noción de objeto empírico o fenómeno, el cual es el resultado de aplicar la síntesis de las categorías a lo dado en la intuición sensible, y donde tanto la determinación de las propiedades como la realidad del objeto son puestas por la constitución subjetiva, y en consecuencia no son independientes del marco conceptual (marco que en el sistema kantiano es único, pues éste no daba cabida a formas alternativas de constituir la realidad). Por otro lado, encontramos la noción de objeto trascendental $=\mathrm{X}$, de un algo indeterminado, general, del que no sabemos ni podemos saber nada ya que no es un fenómeno sino el sustrato de todo fenómeno, pero el cual, sin embargo, no podemos dejar de suponer.

En esta segunda noción de objeto se refleja, a nuestro juicio, el peculiar realismo kantiano. Notemos que es una exigencia de la razón el postular un objeto trascendental, exigencia que obedece - de acuerdo con algunos pasajes de Kant-a la necesidad de que el conocimiento no dé vueltas sobre sí mismo y 
tenga algún amarre en una realidad independiente: "La razón de que no nos baste el sustrato de la sensibilidad y de que añadamos a los fenómenos unos noú menos que sólo el entendimiento puede pensar, se basa en lo siguiente. La sensibilidad y su campo —el de los fenómenos - se hallan, a su vez, limitados por el entendimiento, de forma que no se refieren a las cosas en sí mismas, sino sólo al modo según el cual, debido a nuestra constitución subjetiva, las cosas se nos manifiestan. Tal ha sido el resultado de toda la estética trascendental, resultado que se desprende del concepto de fenómeno en general, a saber, que tiene que corresponder al fenómeno algo que no sea en sí mismo fenómeno. La razón se halla en que éste no puede ser nada por sí mismo, fuera de nuestro modo de representación. Consiguientemente, si no queremos permanecer en un círculo constante, la palabra fenómeno hará referencia a algo cuya representación inmediata es sensible, pero que en sí mismo (...) tiene que ser algo, es decir, un objeto independiente de la sensibilidad" (KRV, A 251-252). Aquí se hace transparente la necesidad conceptual de postular una condición ontológica para evitar que el conocimiento sea un mero juego de representaciones. Por esta vía, entonces, se llega al concepto de una realidad independiente del conocimiento, y por lo tanto externa, a través de un argumento de tipo trascendental.

De acuerdo con la presente interpretación, Kant, a pesar de su fuerte internalismo, debe aceptar la existencia de algo independiente del esquema de categorías, el objeto trascendental, como correlato último de todo conocimiento. Por tanto, habría en Kant dos nociones de realidad y, paralelamente, dos nociones de existencia: (1) la realidad y la existencia como categorías, es decir, como predicados generales que condicionan el fenómeno u objeto empírico, a partir de lo dado en la intuición sensible (y en este sentido, aquello que es real o existente lo sería sólo para nosotros); y (2) la realidad incondicionada de ese algo cuya existencia nos vemos obligados a postular para que nuestro conocimiento no sea tan sólo una ficción de conjunto.

Ahora bien, en algunos pasajes de la Crítica, Kant identifica ese correlato último con la "materia de la experiencia», dándole así un matiz empirista al presupuesto de una realidad incondicionada. En este sentido, encontramos que: "No se puede separar ese objeto trascendental de los datos sensibles, ya que entonces no queda nada por medio de lo cual sea pensado." ( $K R V$, A 250). Y en otro pasaje: "De ahí que lo que en esos objetos [fenómenos] corresponde a la sensación sea la materia trascendental de todos los objetos como cosas-en-sí» (KRV, A 143). Bajo esta óptica, el objeto trascendental parecería ser la materia prima de la expe- 
riencia, la "multiplicidad no sintetizada", a la cual se le da forma vía las intuiciones (espacio y tiempo) y los conceptos del entendimiento (categorías). Entendido así, como sustrato último de los objetos empíricos, se explica que el objeto trascendental sólo pueda ser pensado en relación con la experiencia y los datos sensibles, como afirma Kant en la primera de estas citas. Pero cabe aclarar que cuando decimos "materia prima" no estamos atribuyendo al objeto trascendental ninguna propiedad relacionada con la materia, ya que como el mismo Kant afirma: «El objeto trascendental que sirve de base a los fenómenos externos (...) no es en sí mismo materia ni ser pensante, sino un fundamento - desconocido para nosotros- de los fenómenos que suministran el concepto empírico tanto de la primera [de la materia] como del segundo [del ser pensante]» (KRV, A 379-380).

En nuestra lectura, Putnam estaría muy cerca de esta segunda manera, más empirista, de entender el algo independiente, como se revela en su peculiar caracterización de los objetos empíricos — caracterización que, aparte de sus tintes retóricos, permanece por completo apegada a la noción kantiana de fenómeno. Al aclarar la única noción de objeto empírico que considera admisible, afirma este autor: "los "objetos" mismos son tanto algo que se hace como algo que se descubre, tanto productos de nuestra invención conceptual como del factor "objetivo" en la experiencia, el factor independiente de nuestra voluntad" (Putnam 1981 , p. 54). Como se puede ver, ese factor independiente - al que Putnam se referirá con el término "insumos"- queda ubicado en el nivel de la experiencia; y si bien insiste en que no hay insumos «que no estén ellos mismos, hasta cierto punto, moldeados por nuestros conceptos", de todos modos reconoce-como Kant reconoció- una especie de materia prima de la experiencia, a partir de la cual se conforman los objetos. Cuando "cortamos el mundo en objetos" interactuamos con aquello que, a la vez, posibilita y constriñe las historias - teorías, concepciones del mundo- que podemos inventar o construir. Y es en el reconocimiento de ese factor independiente de nuestra voluntad, donde encontramos el sentido de que Putnam denomine «realismo» al realismo interno.

Por otra parte, Putnam rechaza el realismo metafísico por las mismas razones que Kant, en su momento, rechazara el «realismo trascendental». Su blanco de ataque es el mismo. En la caracterización de Kant: «El realista trascendental interpreta los fenómenos externos (en caso de que se admita su realidad) como cosas en sí mismas, que existen independientemente de nosotros y de nuestra sensibilidad, y que por lo tanto están fuera de nosotros (...)" (KRV, A 369). Putnam, por su parte, comienza por rechazar el supuesto externalista básico que con- 
siste en concebir la realidad como una totalidad de objetos que existen con total independencia de nuestro conocimiento (mente, lenguaje, representaciones o esquemas conceptuales). Y la razón que aduce, en breve, es que: "Los "objetos" no existen independientemente de los esquemas conceptuales" (Putnam 1981, p. 52).

Esta última afirmación genera un mar de confusión cuando no se tiene en cuenta que Putnam está utilizando la noción kantiana de objeto empírico, junto con el sentido de existencia que la acompaña, el de existencia condicionada, que es de hecho el único tipo de existencia involucrado en nuestro conocimiento empírico, sea ordinario o científico. En la perspectiva internalista, entonces, los esquemas conceptuales no serían meros intermediarios entre los sujetos y unos objetos que preexisten a todo saber, como sucede en el externalismo; los esquemas son más bien una pieza clave, indispensable en la constitución misma de los objetos. Los objetos son, por tanto, productos de un proceso de constitución conceptual, proceso que opera desde el nivel mismo de la percepción sensorial.

Ahora bien, a la noción kantiana de objeto, el Putnam historicista le agrega la tesis de la relatividad conceptual, tesis que se podría formular brevemente como afirmando que ningún concepto — ni siquiera las categorías más básicastiene una interpretación única o absoluta. De aquí que la identificación de objetos, que conlleva la atribución de existencia, sea en parte producto de nuestros sistemas de conceptos. Esto ocurre así en todos los ámbitos ontológicos, desde los objetos del sentido común hasta las entidades y procesos de la física teórica. No hay un concepto privilegiado de objeto, ni de existencia, que sea el metafísicamente correcto. La idea de que la realidad nos impone una correspondencia única entre nuestros conceptos y las cosas, como si hubiera una especie de pegamento metafísico entre lenguaje y mundo, es una mera ilusión. El fenómeno de la relatividad conceptual «depende del hecho de que los mismos primitivos lógicos, y en particular las nociones de objeto y existencia, tienen una multitud de usos diferentes, y no un "significado" "absoluto" (Putnam 1987, p. 19).

Así entendida, la tesis de la relatividad conceptual trae consigo un pluralismo ontológico, pues abre la posibilidad de tener concepciones del mundo con ontologías distintas -incluso incompatibles - que resulten igualmente adecuadas en ciertos contextos, en función de determinados intereses y objetivos (considérense, por ejemplo, los casos de teorías científicas empíricamente equivalentes pero ontológicamente incompatibles). De aquí que la pregunta sobre qué es lo que hay en el mundo, requiera de la especificación del esquema conceptual, len- 
guaje o teoría, donde se plantea e intenta responder. Sólo cuando hemos adoptado un sistema de conceptos podemos afirmar que, en efecto, algunos hechos y objetos están ahí para ser descubiertos. En otras palabras, sólo desde el plano del conocimiento tiene sentido formular preguntas ontológicas. Pretender lo contrario sería cometer el error de poner la carreta de la metafísica delante del caballo de la epistemología. De esto se desprende que la crítica de fondo al realismo metafísico, externalista, se dirija contra su compromiso con categorías ontológicas absolutas. La tesis externalista de que ciertos enunciados, los verdaderos, describen el mundo de una manera que es independiente de toda perspectiva conceptual, supone el compromiso con categorías que sólo podrían ser las del punto de vista del Ojo de Dios.

Tenemos entonces que la noción internalista de objeto tiene, cual nuevo Jano, una doble cara. Por una parte, los objetos no son meras invenciones libres de la mente, sin un sustrato independiente que impone ciertas restricciones; pero, por otra parte, los objetos tampoco son cosas puramente externas, dadas por sí mismas, con propiedades esenciales y relaciones intrínsecas. Pensar en términos de esta alternativa sería pensar en términos de una falsa dicotomía. Los objetos son, a la vez, productos de la mente y del mundo. La mente no se limita a copiar un "mundo ya hecho", pero tampoco es la mente la que hace al mundo. De aquí la famosa frase de Putnam de que "la mente y el mundo hacen conjuntamente a la mente y al mundo» (Ibid., p. xi).

A su vez, esta mancuerna mente-mundo nos conduce a otra de las claves de la perspectiva internalista, el desvelamiento de la extendida «falacia de la división" que Kant șeñalara por primera vez. Esta falacia consiste en creer que podemos distinguir, en nuestra experiencia, entre aquello que nosotros aportamos -nuestra propia contribución conceptual- y aquello que forma parte del mundo tal como es en sí mismo. El error de esta pretensión, característico del realismo trascendental o externalista, consiste en interpretar los fenómenos físicos, las cosas materiales, como objetos que existen independientemente de nuestra sensibilidad pero que a la vez nos son epistémicamente accesibles. En suma, se supone como cognoscible algo que por definición es incognoscible.

Para concluir este examen del realismo internalista, podríamos decir que a pesar de que se haya abandonado el carácter único que Kant otorgara al esquema de categorías, y en su lugar se defienda ahora la existencia de esquemas conceptuales alternativos, no convergentes ni reducibles a un esquema único, de todos 
modos siguen vivas las intuiciones kantianas más básicas a través de sus desarrollos pluralistas. En la perspectiva internalista se sigue afirmando que "las cosas para nosotros" son simplemente las cosas, que no hay "detrás" de los objetos de nuestro conocimiento otros objetos (noúmenos) que son los que realmente existen. Los objetos de los que hablamos desde nuestras estructuras conceptuales no son meras proyecciones, ilusiones o apariencias, sino que son los únicos objetos que en tanto objetos realmente existen.

Por otra parte, contra lo que muchos piensan, el reconocimiento elemental de que "la mente humana no ha creado las estrellas ni las montañas" no basta para dirimir la discusión entre externalistas e internalistas, fallando a favor de los primeros (Cf. Putnam 1990, p. 30). Este reconocimiento simplemente expresa un compromiso realista indispensable, casi diriamos trivial, que se opone a un idealismo que afirmara que sólo existe lo mental, o que todo lo que existe es un mero producto del pensamiento. A nuestro juicio, ninguna concepción que hoy en día afirmara tal cosa merecería la pena de ser discutida (al examinar la posición de Kuhn, más adelante, retomaremos la cuestión de la realidad independiente).

Antes de Kant, la polémica giraba en torno de si hay realmente objetos «ahí afuera", objetos físicos o materiales, o si sólo existe lo mental (por ejemplo, Locke versus Berkeley). Kant comienza por rechazar los supuestos de esta discusión entre "realistas trascendentales" e "idealistas empíricos", como él los llamaba, y plantea el problema del realismo en otros términos. No se trata de establecer qué es lo que realmente existe, el problema está en aceptar que todo aquello que llamamos «objeto", del tipo que sea, está constituido dentro de algún esquema conceptual y es, por tanto, "objeto para nosotros». De aquí que todo conocimiento sólo pueda ser conocimiento de fenómenos.

Si esto es así, el verdadero contrincante a partir de Kant es el externalista, sea materialista, idealista o dualista. Esto es, todo aquél que insista en suponer categorías ontológicas absolutas, en distinguir lo que es en sí de lo que es sólo para nosotros (cometiendo la falacia de la división), en suponer que el mundo nouménico es el responsable del valor de verdad de nuestros juicios empíricos $y$, en consecuencia, aquél que siga preguntando, a secas, qué es lo que realmente existe. Si no postulamos alguna facultad como la intuición intelectual que suponían los filósofos medievales, que nos permita un acceso no mediado a ciertos aspectos del mundo, no podemos pretender que existe alguna forma de referirse a objetos o hechos empíricos que sea independiente de nuestros sistemas conceptos. 
Y si bien, como afirma Putnam: «Quizá Kant fue demasiado ambicioso al pensar que podía especificar los constreñimientos a priori del proceso de construcción (...) [sin embargo] la idea de que toda experiencia implica construcción mental, y la idea de que la dependencia entre los conceptos de objetos físicos y los conceptos de experiencia va en ambos sentidos, continúan siendo de gran importancia en la filosofía contemporánea» (Ibid., p. 210).

\section{Kant, Kuhn y la pluralidad de mundos}

Con base en sus análisis de la dinámica evolutiva de la ciencia, Kuhn intentaba convencernos de que no hay evidencia histórica que apoye la especulación de que el desarrollo científico converge hacia una teoría o concepción última del mundo. Si bien en disciplinas como la física se pueden rastrear series de ecuaciones cada vez más exactas en la predicción de cierto tipo de fenómenos, lo importante para esta discusión es que las concepciones sucesivas, utilizadas para interpretar dichas ecuaciones, han sido trastocadas una y otra vez por cambios profundos, cambios donde se modifican los compromisos con las entidades o mecanismos que se postulan como factores explicativos. Así, la concepción teórica de la mecánica newtoniana fue fundamentalmente modificada por la de la relatividad general, y por una especie de inducción histórica bien podemos esperar que esta última sea globalmente reemplazada por la teoría de la supergravedad, o por alguna otra concepción aún no imaginada. Como afirma Kuhn: «La comparación de teorías en la historia no da lugar para pensar que sus ontologías se aproximan hacia un límite: en ciertos aspectos fundamentales, la teoría general de la relatividad de Einstein se parece más a la física de Aristóteles que a la de Newton» (Kuhn 1970, p. 265). De aquí que al efectuar esta comparación histórica nos encontremos más bien con una serie de concepciones que suponen ontologías o "mundos" divergentes.

Ya desde La estructura de las revoluciones cientificas, capítulo X, se perfilaba el problema ontológico de fondo, el problema del cambio de mundos, que traían consigo las tesis kuhnianas sobre la dinámica científica: "Aunque el mundo no cambia con un cambio de paradigma, el científico trabaja después en un mundo diferente» (Kuhn 1962, p. 121). Y acto seguido Kuhn insiste -a sabiendas de que está planteando un enigma de carácter filosófico- en que tenemos que encontrar la manera de dar sentido a afirmaciones como ésta. 
Lo primero que salta a la vista es el doble uso de la palabra «mundo». Por un lado se afirma que "después de una revolución el científico sigue mirando el mismo mundo", pero por otro lado se sostiene que "cuando cambian los paradigmas, el mundo mismo cambia con ellos». ¿Cómo conciliar entonces ambas intuiciones? A nuestro modo de ver, remitiendo directamente estos dos sentidos de mundo, "el mundo que no cambia" y "el mundo que sí cambia", a los dos sentidos de realidad - y de existencia - que se encuentran en el pensamiento de Kant: por un lado, la realidad que existe con total independencia del esquema de categorías, esto es, el objeto trascendental; y por el otro, el mundo de la experiencia, es decir, el mundo de objetos empíricos cuya existencia está condicionada por dicho esquema.

Pero antes debemos subrayar que el problema del cambio de mundos sólo se plantea cuando se toma en serio la tesis de inconmensurabilidad, esto es, cuando se reconoce que no todas las descripciones de un dominio de investigación son traducibles o formulables en el lenguaje de paradigmas alternativos. A partir de los años 80, cuando Kuhn desarrolla la formulación taxonómica, se pone en claro que la inconmensurabilidad implica una divergencia en la estructura de categorías ontológicas: dos teorías son inconmensurables cuando sus estructuras taxonómicas no son homologables (Cf. Kuhn 1981). Y es precisamente este hecho - la divergencia en la manera de clasificar o agrupar las entidades de un dominio de investigación - lo que en última instancia daría cuenta de la imposibilidad de reducir los diversos mundos, los diversos recortes ontológicos, a uno solo. De aquí que, en nuestra lectura, la formulación taxonómica de la inconmensurabilidad no haya hecho más que reforzar la perspectiva internalista y pluralista de Kuhn, así como su fuerte filiación con una tradición kantiana. Veamos esto con más detenimiento.

$\mathrm{Al}$ analizar el proceso de aprendizaje de un léxico - de un vocabulario estructurado- Kuhn sostiene que en este proceso se adquieren las categorías taxonómicas que permiten describir el mundo de cierta manera. Pero no sólo eso. También afirma que las categorías traen consigo, de manera inseparable, el mundo en el cual viven los miembros de una comunidad lingüística. Esto es, cuando las estructuras léxicas de dos comunidades no son homologables, es decir, cuando sus concepciones del mundo son inconmensurables, «algunas de las clases que pueblan [sus] mundos son irreconciliablemente diferentes, y la diferencia ya no es más entre descripciones sino entre las poblaciones que se describen" (Kuhn 1993, p. 319; énfasis nuestro). Con lo cual se pone claramente de 
manifiesto que las categorías taxonómicas, además de descriptivas, son constitutivas de las entidades que pueblan los respectivos mundos. Y si esto es así, Kuhn queda básicamente comprometido con una noción kantiana de objeto empírico, distintiva de la perspectiva internalista.

En este mismo derrotero, cabe destacar que Kuhn retoma la distinción que hiciera Hans Reichenbach entre un a-priori fijo (el del carácter absoluto de las categorías kantianas) y un a-priori relativo a las distintas culturas y períodos históricos. Y es justamente este segundo sentido de a-priori el que Kuhn otorga a las categorías taxonómicas, ya que a pesar de no ser fijas, de todos modos son constitutivas de la experiencia posible del mundo (Cf. Ibíd., p. 331). De este modo, si el mundo de la experiencia depende de manera constitutiva de los sistemas de categorías, tenemos entonces que el repertorio de entidades que se consideran existentes no puede permanecer inmutable a través del cambio de paradigmas (del cambio de estructuras taxonómicas). La diversidad de mundos, en consecuencia, no es sólo una metáfora o un mero recurso retórico, sino que implica una genuina pluralidad ontológica.

En cuanto al «mundo que no cambia», encontramos que cuando Kuhn se pregunta por la relación entre la taxonomía que comparte una comunidad y el mundo que esa comunidad habita, responde sin vacilar que esa relación no se puede entender a la manera del realismo metafísico, ya que: "En la medida en que la estructura del mundo puede ser experimentada [...] dicha estructura está constreñida por la estructura del léxico de la comunidad que lo habita» (Kuhn 1991, p. 10). Y cabe señalar que la estructura de un léxico, además de estar biológicamente condicionada, depende también de los distintos procesos de socialización y educación profesional.

Sin embargo, cuando Kuhn se da cuenta de que estas afirmaciones suyas podrían sugerir la idea de que lo que llamamos «mundo real» es dependiente de la mente, reacciona de manera tajante y defensiva, puesto que interpreta dicha dependencia en el sentido aberrante de que el mundo es una mera invención o construcción mental. Kuhn quiere dejar claro, como cualquier internalista sensato, que los sujetos "ya encuentran el mundo en su sitio", que el mundo real es "el escenario de toda vida individual y social», y que ese mundo, tan nos impone restricciones, que la supervivencia sólo es posible si los sujetos se adaptan a ellas. "¿Qué más se puede razonablemente pedir de un mundo real?" (Ibídem), se pregunta Kuhn. 
Aquí es importante decir que la referencia a las restricciones que nos impone el mundo real no significa, de manera alguna, que Kuhn esté cometiendo la falacia de la división y pretenda que podemos distinguir nuestra contribución conceptual de aquello que nos impone el mundo tal cual. En este respecto, en clara analogía con la evolución biológica, Kuhn supone una especie de "plasticidad mutua» entre los sujetos y el mundo, plasticidad que impediría trazar una línea divisoria entre el mundo real y el mundo descrito o representado (el cual es producto de nuestra interacción con el mundo real). Si lo que de hecho evoluciona es la conjunción del nicho y las criaturas que lo habitan, para poder hablar de adaptación no es necesario "trazar una línea entre las criaturas dentro del nicho y su entorno "externo" (Ibíd., p. 11). Como se puede ver, Kuhn podría haber dicho al unísono con Putnam que "la mente y el mundo hacen conjuntamente a la mente y al mundo".

Otra afinidad con el Putnam internalista la encontramos en que Kuhn habla del mundo independiente como algo «experiencialmente dado", que "no es para nada respetuoso de los deseos de un observador" y que "es muy capaz de proporcionar evidencia en contra de las hipótesis que fracasan en ajustarse a su comportamiento" (Cf. Ibíd., p. 10). Ya en sus primeros escritos decía que «no se puede forzar a la naturaleza a entrar en un conjunto arbitrario de cajas conceptuales» (Kuhn 1970, p. 263). Pero también sostenía que, como nos enseña la historia, seguramente la naturaleza no se quedará confinada indefinidamente en ninguna de las estructuras conceptuales que los científicos hayan construido hasta ahora. Recordemos que fue Kuhn quien puso las bases del argumento de la "metainducción pesimista» -que más tarde desarrollara Larry Laudan (1981) - en contra del realismo científico. Y cabe señalar que como agudo crítico de este tipo de realismo, Kuhn también objeta la pretensión de que podemos distinguir entre propiedades esenciales (imtrínsecas) y propiedades secundarias (aparentes), dando una serie de argumentos contra la teoría causal de la referencia, con el fin de mostrar que el valerse del vocabulario científico más desarrollado - como en la línea trabajada por $S$. Kripke- tampoco mejora la situación del realismo metafísico en su versión cientificista. $\mathrm{Al}$ argumentar que la referencia de los términos es una función de la estructura léxica, Kuhn observa en una nota a pie de página que: «quienes sostienen que la referencia es independiente del significado también sostienen que la metafísica es independiente de la epistemología (Kuhn 1990, p. 317, nota 22), independencia que desde luego rechaza como buen internalista. 
Recapitulando, las anteriores afirmaciones revelan los siguientes rasgos del realismo kantiano que asume Kuhn. En primer lugar, la idea del mundo como algo "experiencialmente dado" revela su acuerdo con la versión más empirista del supuesto del algo independiente, esto es, como sustrato o materia de la experiencia. Se trata de un mundo que opone resistencia a través de los insumos de la experiencia, que serían el factor objetivo e independiente de nuestra voluntad, por más que no haya insumos que no estén contaminados o moldeados por nuestros sistemas de conceptos. En este respecto, recordemos que Kuhn abrió brecha como uno de los principales defensores de la famosa tesis de la "carga teórica de la observación».

También se destaca el supuesto de que el mundo tiene alguna estructura, aunque este supuesto viene acompañado de la convicción de que ni siquiera de nuestras teorías científicas más exitosas podemos aseverar un isomorfismo con dicha estructura. Por una parte, el hecho de que la naturaleza no encaje en cualquier estructura conceptual -y en consecuencia no cualquier teoría resulte aceptable - permite suponer que el mundo tiene alguna estructura. Pero por otra parte, el argumento de la subdeterminación de las teorías por la evidencia (que permite tener teorías empíricamente equivalentes pero ontológicamente incompatibles), aunado al poderoso argumento de la metainducción pesimista, ponen seriamente en tela de juicio cualquier pretensión de establecer una correspondencia estricta entre nuestras teorías y la realidad independiente.

Si bien es cierto que queda como un punto ciego cómo se articularía el supuesto de que el mundo tiene alguna estructura con las tesis internalistas más básicas - articulación que subsiste como un gran desafío desde los tiempos de Kant-, sin embargo se podría decir que, al menos en principio, este supuesto no parece incompatible con un realismo de tipo interno. Después de todo, como afirma Quine (con base en el argumento de la inescrutabilidad de la referencia), lo único que nuestra ciencia necesita es que el mundo esté estructurado de tal manera que acredite nuestras predicciones, y ni siquiera en el ámbito de la ciencia podemos plantear exigencias más concretas acerca del mundo (Cf. Quine 1981, p. 32).

Al final de «El camino desde La estructura», donde se autocataloga como un kantiano neo-darwinista, Kuhn sintetiza su manera de ver la relación entre nuestro conocimiento y el mundo, síntesis que podría leerse como una declaración 
de su acuerdo básico con un realismo internalista. Lo citamos extensamente en apoyo de la interpretación aquí propuesta: "Subyaciendo a todos estos procesos de diferenciación y cambio [de categorías taxonómicas] debe haber, por supuesto, algo permanente, fijo y estable. Pero, como la Ding an sich de Kant, es inefable, indescriptible, indiscutible. Situada fuera del espacio y del tiempo, esta fuente kantiana de estabilidad es el todo a partir del cual han sido generados tanto las criaturas como sus nichos, tanto los mundos "internos" como los "externos". La experiencia y la descripción sólo son posibles con el descriptor y lo descrito separados, pero la estructura léxica que marca esa separación puede hacerlo de diversas formas, resultando cada una de ellas en una forma de vida diferente, aunque nunca completamente diferente. Algunas formas están mejor adaptadas a ciertos propósitos, y otras formas a otros. Pero ninguna debe ser aceptada como verdadera o rechazada como falsa; ninguna nos ofrece un acceso privilegiado al mundo real" (Kuhn 1991, p. 12).

\section{En defensa de la racionalidad acotada.}

Buena parte de los problemas que llevamos discutidos suponen un complemento reflexivo muy adecuado para situar las discusiones contemporáneas sobre la noción de racionalidad y los agentes epistémicos.

Un agente epistémico que se considerase capaz de acceder al mundo en sí (un realista externalista), no tendría en cuenta ninguna de las constricciones de tiempo, información y capacidades computacionales que, inevitablemente, tenemos los agentes humanos. Ese agente ideal, caso de existir, tendríamos que hacerlo residir en el Olimpo. Se trata en este caso de una idealización infructuosa, aunque no olvidemos que siempre trabajamos con modelos y con idealizaciones.

Frente a esos agentes olímpicos parece razonable plantear como modelo ideal un sujeto situado en el mundo e interrelacionado con otros agentes, que sortea sus propias limitaciones temporales, informativas y computacionales en su interacción con el mundo y con otros agentes. La expresión de su autonomía se da precisamente en esa capacidad de interacción y de utilizar reglas de decisión rápidas, poco "costosas" desde el punto de vista informativo, que se corresponden con sus teorías y con la forma en que esas teorías permiten el acceso al conocimiento. 
Este otro tipo de agente epistémico se conecta bien con la idea de racionalidad acotada de H. Simon, quien utilizaba la metáfora de la tijera para señalar nuestras limitaciones internas, computacionales, y las que vienen dadas por el entorno - otra vez podríamos decir que «la mente y el mundo hacen conjuntamente a la mente y al mundon. En una de las hojas de la tijera de Simon se sitúan las limitaciones cognitivas de los seres humanos, mientras que en la otra se encuentra la estructura del entorno: la racionalidad cognitiva y la racionalidad ecológica, como en la actualidad las llama G. Gigerenzer. Aunque lo importante en este respecto es que las mentes con tiempo, conocimiento y demás recursos limitados pueden, a pesar de todo, tener éxito a la hora de explotar la estructura de sus entornos (Cf. Selten 2001, p. 39).

Incrementar la complejidad de una tarea no necesariamente implica incrementar de manera correspondiente la complejidad de los individuos. A veces una mejor comprensión del entorno puede ayudar a resolver la tarea. Un sistema de relaciones determinado permite a veces la adopción de un mecanismo rápido y sencillo, el cual produce mejores resultados que los que supone la racionalidad óptima que se presenta con un alto grado de complejidad computacional. Algunos movimientos hacia una mayor comprensión de la génesis misma de esas reglas en la interacción con el mundo, pueden ser considerados parte de la clave de nuestra epistemología de agentes acotados. Aceptar que razonamos en un proceso de constante interacción con el mundo es el aspecto central compartido entre los defensores de la acción situada y los de la cognición distribuida.

Aunque cierta línea de trabajo asentada en el ámbito de la psicología evolutiva y de los estudios cognitivos considera que tenemos diferentes tipos de "inteligencia», o de capacidades cognitivas, que además están asociadas con determinadas situaciones especializadas (sentido social, física ingenua, capacidades numéricas, etc.), sin embargo hay quienes han caracterizado la peculiaridad de la capacidad cognitiva humana como una especial capacidad de comunicación e integración de los diversos tipos de habilidades. Cabe considerar en serio la hipótesis de que el principal logro cognitivo, definitorio del conocimiento humano, sea precisamente el pensamiento integrado.

Desde esta posición, la caracterización de la acción racional, como peculiar de los humanos, requiere salirse del modelo estándar que establece disociativamente la estructura formal del conocimiento como su elemento más característico. Por ello defendemos una teoría sintética de la racionalidad 
como herramienta conceptual mínima y necesaria para acercarnos al estudio de la ciencia. La dificultad con el modelo estándar de la racionalidad no sólo consiste en que se refiera a sujetos que, en su momento, A. Sen (1977) tildara de «imbéciles racionales", o en que sea un reflejo de cierta forma de hiperracionalismo, como el propuesto en Racionalidad y accion bumana por Jesús Mosterín. El principal problema con el modelo estándar, a nuestro modo de ver, es que no logra representar idealmente uno de los principales rasgos de la acción humana, esa capacidad integrativa, la capacidad de equilibrio reflexivo, que conjuga elementos situacionales, emocionales y lógicos. Desde luego, en esta dirección podríamos apoyarnos en trabajos como los de A. Damasio (por ejemplo, Damasio 1999).

Ahora bien, el cambio más importante en esta línea consiste en considerar que ese proceso de integración no se da en el vacío sino que es el resultado de una interacción con el mundo, interacción que viene a ser el principio fundamental de lo que se conoce como actividad situada y cognición distribuida. Una cierta visión caricaturizada de los modelos cognitivos tradicionales nos muestra la cognición como la construcción de modelos internos del mundo, en el cerebro de los individuos, quienes a partir de ahí planifican sus acciones. La cognición distribuida plantea que la cognición viene a ser una propiedad emergente de la interacción entre grupos de personas y su entorno. En todo caso, como indica Alan Dix en "Imagination and Rationality" (2003), es cierto que algunos modelos más tradicionales de la cognición también trataban de modelar ese modo interactivo de pensar.

Siguiendo en parte a A. K. Sen, consideraremos a nuestros conceptos como filtros que seleccionan la información de entre toda la disponible; y es precisamente aquella información, una vez filtrada, la que deviene información pertinente para nosotros. Así las cosas, resulta fundamental la perspectiva o posición, junto con el esquema conceptual implicado, desde donde recogemos la información; la objetividad humanamente posible es, siempre, relativa a la posición (positional objectivity).

Un primer paso para avanzar en la construcción de un modelo más adecuado de la racionalidad humana, consiste en admitir que la objetividad no aparece como aquel punto de vista que resulta independiente de cualquier posición o esquema conceptual, sino que siempre es el resultado de un punto de vista centrado en un lugar particular. En vez de considerar la objetividad como the view from 
nowhere, a la manera externalista, es conveniente entenderla en clave internalista, es decir, como the view from a delineated somewhere (Cf. Sen 1993, p. 127).

Adoptar la idea de filtro informativo para nuestros juicios empíricos, como hace Sen, es un buen primer paso. Sin embargo, habría que decir que esa idea retiene cierto carácter pasivo, en tanto que el filtro aparece a veces como una mera criba que se limita a dejar pasar unas cosas e impedir que pasen otras. De aquí la conveniencia de extender dicha noción. Pero también debemos reconocer que ya el simple hecho de atender a la capacidad de filtraje que tienen nuestros conceptos nos pone, de entrada, en la dirección de atender a los componentes pragmáticos de nuestra teoría de la racionalidad. De aquí que para desarrollar esta extensión podríamos utilizar una metáfora diferente, que pudiera servir como herramienta adicional para la teoría de la racionalidad. Esta vez el préstamo metafórico lo vamos a extraer de un ámbito más cercano a la biología y a la química, que a la física. Se trata de la noción de membrana semipermeable.

Consideramos que pensar en nuestro modelo de ser humano como cierto tipo de membrana semipermeable puede ser más eficaz que entendernos como meros filtros selectores de información. La membrana semipermeable es «sensible al contexton, por así decirlo. La membrana tiene una capacidad de filtraje que depende de la concentración de la solución en cuyo seno actúa. Incluso, dando un paso más, tanto las membranas como los filtros pueden verse englobados en una red o tejido. Una membrana, según el diccionario de la lengua, es una capa delgada de tejido orgánico, elástica y resistente que separa dos cavidades o envuelve algún órgano. El filtro nos remite a una materia porosa -por ejemplo, cierto papel- $\mathrm{o}$ a un dispositivo de cualquier clase que sirve para colar; se aplica también a otros dispositivos destinados a dejar pasar parte de una cosa y retener otra parte. Proponemos entonces una trilogía conceptual (filtros, membranas y tejidos-redes) con la cual pensar críticamente a propósito de la relación entre la ciencia y el mundo. Serían, por así decirlo, las piezas mínimas para construir un modelo de agente epistémico que supere algunas de las conocidas deficiencias del elector racional.

Una rancia tradición filosófica parece decirnos que no se puede pasar del ser al deber ser. De cómo son las cosas a cómo deberíamos comportarnos. Sin embargo, tal parecería que tanto la opción ética como otros componentes axiológicos resultan ser previos, en muchos casos, sirviéndonos por ello como filtros de 
información (incluso reflexivamente, esto es, al elaborar la misma noción de objetividad relativa a la posición). Si no se tiene en cuenta, desde un principio, cierto tipo de información, no se la podrá incorporar posteriormente; ya ha quedado fuera, relegada, en el proceso de idealización o abstracción. A nuestro parecer, un problema de este tipo es el que ha señalado Marcelo Dascal a propósito de la obra de Popper, al decir que el racionalismo crítico aparece con demasiada frecuencia como racionalista pero sin "críticos" -sin "críticos de carne y hueso" (Cf. Dascal 2001).

En definitiva, nos parece razonable seguir ahondando en el camino que lleva de Kant a Kuhn, ya que en esa senda podemos encontrar elementos para construir un mejor modelo de agente epistémico, más adecuado a nuestras limitaciones humanas. Comprender mejor nuestras limitaciones es un camino más que conveniente para ampliar nuestras propias capacidades de acción como críticos racionales.

\section{bibliografía}

Damasio, A. (1999), The Feeling of What Happens: Body and Emotion in the Making of Consciousness, Harcourt Brace, New York.

DASCAL, M. (2001), "Reputation and refutation: Negotiating Merit", en Weigand, E. y M. Dascal (comps.), Negotiation and Power in Dialogical Interaction, J. Benjamins Publishing Company, Amsterdam, pp. 3-17.

DIx, A. (2003), Imagination and Rationality, http://www.hcibook.com

GIGERENZER, G. y R. SELTEN (comps.) (2001), Bounded rationality: the adaptive toolbox, MIT Press, Cambridge, Mass.

KANT, I. (1781), Kritik der reinen Vernunft, (versión inglesa Critique of Pure Reason, traducción de N. Kemp Smith, St. Martin's Press, Nueva York, 1965 y versión española con Prólogo, notas e índices de Pedro Ribas, Ediciones Alfaguara, Madrid, 1978).

KuHN, T.S. (1962), The Structure of Scientific Revolutions, University of Chicago Press, 2a. ed. aumentada, 1970; v.e. La estructura de las revoluciones cientificas, Fondo de Cultura Económica, México, 1971 (se cita de la edición inglesa).

KUHN, T.S. (1970), «Reflections on my Critics», en I. Lakatos y A. Musgrave (eds.) (1970), pp. 231-278; v.e. "Consideración en torno a mis críticos», pp. 391-454.

Kunn, T.S. (1981), "What are Scientific Revolutions?», en L. Krüger, L.J. Daston y M. 
Heidelberger (eds.), The Probabilistic Revalution, MIT Press, Cambridge, 1987, pp. 7-22; v.e. "¿Qué son las revoluciones científicas?" en ¿Qué son las revoluciones cientificas? y otros ensayos, Paidós, 1989.

KuHN, T.S. (1990), "Dubbing and Redubbing: the Vulnerability of Rigid Designation", en W. Savage (ed.), Scientific Theories. Minnesota Studies in the Philosophy of Science, Vol. XIV, University of Minnesota Press, Minneapolis, pp. 298-318.

KuHN, T.S. (1991), "The Road Since Structure», en A. Fine, M. Forbes y L. Wessels (eds.): PSA 1990, Vol. 2, Philosophy of Science Association, East Lansing, pp. 3-13; v.e. "El camino desde La estructura»: Arbor, Vol. CXIVIII, No. 583, pp. 27-46 (se cita del original).

KuHN, T.S. (1993), "Afterwords", en P. Horwich (ed.), World Changes. Thomas Kubn and the Nature of Science, The MIT Press, Cambridge, Mass., 1993, pp. 311-341.

LAKATOS, I. (1971), "La historia de la ciencia y sus reconstrucciones racionales", en la v.e. aumentada de I. Lakatos y A. Musgrave (eds.) (1970), pp. 455-509.

LaKaTos, I. y A. Musgrave (eds.) (1970), Criticism and the Growth of Knowledge, Cambridge University Press, Londres; v.e. aumentada La critica y el desarrollo del conocimiento, Grijalbo, Barcelona, 1975.

LaUdan, L. (1981), "A confutation of convergent realism": Philosophy of Science, No. 48, pp. 19-49.

Pérez Ransanz, A.R. (1998), "El cambio de mundos y el realismo», en C. Solís (comp.), Alta tensión: Historia, filosofia y sociologia de la ciencia, Paidós, Barcelona, pp. 259-277.

Pérez Ransanz, A.R. (1999), Kubn y el cambio cientifico, FCE, México.

Putnam, H. (1981), Reason, Truth and History, Cambridge University Press, Cambridge; v.e. Razón, verdad e historia, Tecnos, Madrid, 1988.

PutNam, H. (1987), The Many Faces of Realism, Open Court, La Salle, Illinois; v.e. Las mil caras del realismo, Paidós, Barcelona, 1994.

PUTnam, H. (1990), Realism with a Human Face, Harvard University Press, Cambridge, Mass.

QUINE, W.V.O. (1981), Theories and Things, The Belknap Press of Harvard University Press, Cambridge, Mass.; v.e. Teorias y Cosas, UNAM, México, 1986 (se cita de la v.e.).

SELTEN, R. (2001), "What Is Bounded Rationality?" en Gigerenzer, G. y R. Selten (comps.), Bounded rationality: the adaptive toolbox, MIT Press, Cambridge, Mss., pp. 37-51. 
SEN, A. K. (1977), «Rational Fools: A Critique of the Behavioral Foundations of Economic Theory": Philosophy and Public Affairs, 6, 317-344.

SEN, A.K. (1993), "Positional Objectivity», Philosophy and Public Affairs, 22, 126-145. VAN FraASSEN, B.C. (1989), Laws and Symmetry, Clarendon Press, Oxford. 